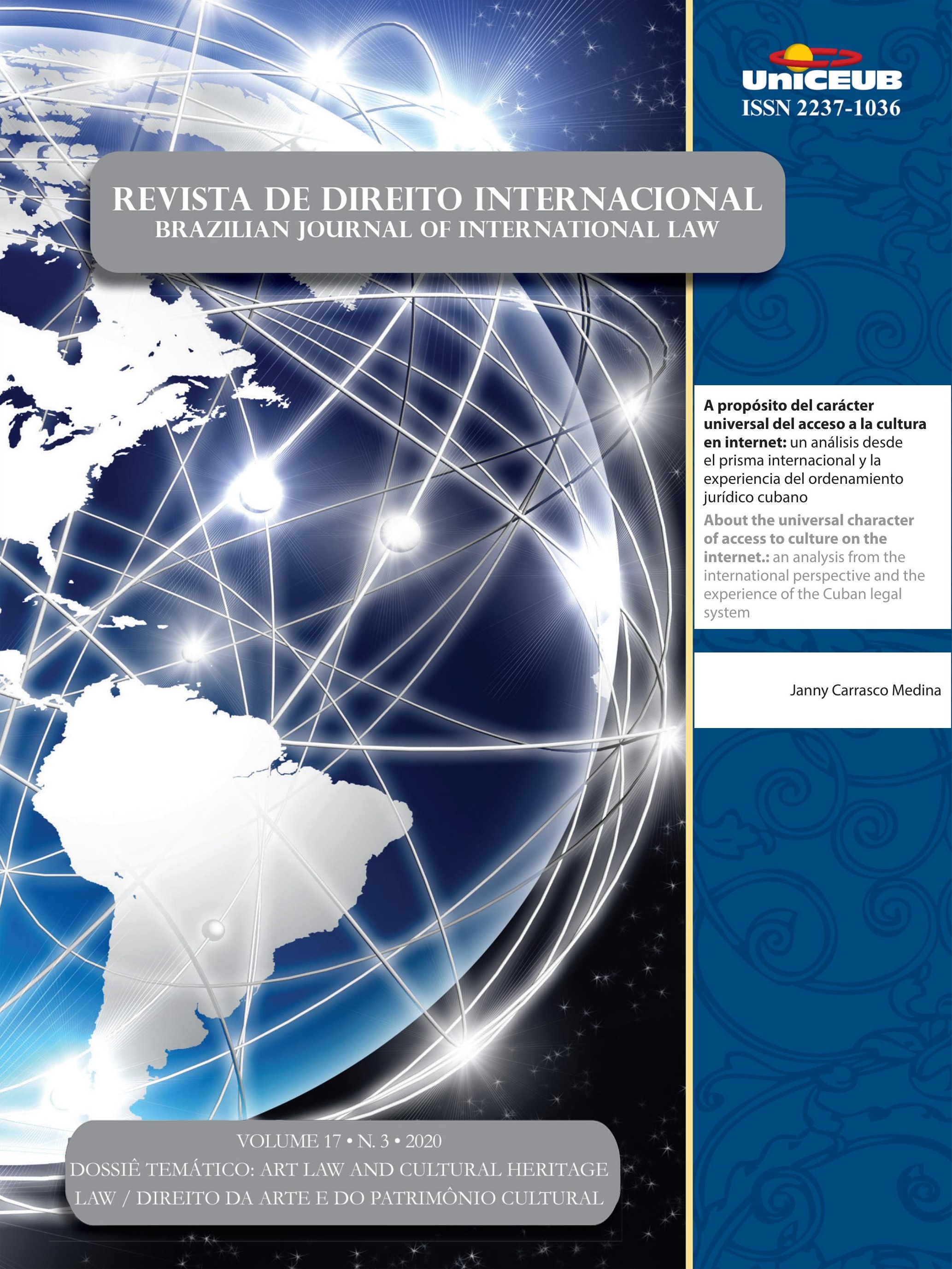




\section{Sumário}

EDITORIAL .20

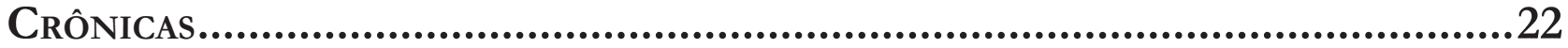

O COSTUME INTERNACIONAL COMO REFORÇO DA OBJEÇÃO BRASILEIRA À CLÁUSULA DO TRATAMENTO JUSTO E EQUITATIVO .24

Leonardo Vieira Arruda Achtschin

O PROCESSO LEGISLATIVO COMO GARANTIA PARA A OBTENÇÃo DO CONSENTIMENTO PRÉVIO DAS COMUnidades quilombolas de AlCÂNTARa

Gabriel de Oliveira Borba

Dossiê temático: Art Law and Cultural Heritage law / Direito da Arte e do Patrimônio cultural - Panorama Geral

Peoples' heritage or States' heritage? sovereignty in the UNESCO mechanism for THE SAFEGUARDING OF INTANGIBLE CULTURAL HERITAGE.

Aliki Gkana

The IMPACT OF THE UNESCO AND UNIDROIT CONVENTIONS AND THE EU DIRECTIVES ON THE INTERNATIONAL ART MARKET: AN ANALYSIS FIFTY YEARS AFTER THE INTRODUCTION OF THE OBLIGATION TO RETURN STOLEN OR ILLEGALLY EXPORTED CULTURAL GOODS 61

Geo Magri

Três pautas em destaque na agenda de diversidade Cultural da Unesco: Ambiente digiTAL, TRATAMENTO PREFERENCIAL E PARTICIPAÇÃO DA SOCIEDADE CIVIL............................76

Danilo Júnior de Oliveira, Maria Carolina Vasconcelos Oliveira e Ana Paula do Val

A 100 YEARS INSTITUTIONALIZED CULTURAL HERITAGE PROTECTION: FROM THE INSTITUTIONALIZED INTERNATIONAL COOPÉRATION INTELLECTUELLE TO THE HUMAN RIGHT TO CULTURAL HERITAGE

Lando Kirchmair 


\section{Aspectos Metodológicos do Direito da Arte e do Patrimônio}

Cultural

A proteção do PATrimônio CULTURAL EM NOVAS PERSPECTIVAS: ESTUdo COMPARAdo ENTRE A Kulturgutschutzgesetz e a Holocaust Expropriated Art Recovery Act of 2016.....111 Ardyllis Alves Soares

ArT-RELATED DispUTES AND ADR METHODS 127 Maria Beatrice Deli e Veronica Proietti

Due Diligence in Art Law and Cultural Heritage Law 150 Lisiane Feiten Wingert Ody

The Reception of Droit de Suite in International Law: Diagnosis and Remedy .... 170 Mickael R. Viglino

Direito da Arte e do Patrimônio Cultural: do Regional ao Local ....... 188

Câmara Cascudo e o legal Design - A Visualidade do Direito entre Provincianismo e GlobalizaÇão 190 Marcilio Toscano Franca Filho

A política da União Europeia no turismo: O turismo cultural e a sustentabilidade do PATRIMONNIO INDUSTRIAL PARA INTEGRAÇÃO DO BLOCO EUROPEU

Maraluce Maria Custódio e Fernando Barotti dos Santos

Diálogo entre la Corte Interamericana de Derechos Humanos y el Tribunal Europeo de Derechos Humanos en torno al Derecho humano a la identidad cultural..223 Juan Jorge Faundes

Digital ART AND THE BELT AND ROAD INITIATIVE: CHALLENGES AND OPPORTUNITIES 257 Dan Wei e Ângelo Rafael

Policing heritage crime in Latin America. .275 Naomi Oosterman e Donna Yates 
The principles of Cultural Heritage Law based on the Polish Law as an example.292 Małgorzata Joanna Węgrzak e Kamil Zeidler

Heritage Protection in INTERNATIONAL LAw AND NATIONAL LAW: INSIGHTS INTO THE CASE OF VIETNAM

Yen Thi Hong Nguyen e Dung Phuong Nguyen

THE APPROPRIATION OF THE CARIOCA INTANGIBLE CULTURAL HERITAGE BY AN ENTREPRENEURIAL LOGIC

Mário Ferreira de Pragmácio Telles

A Propósito del CARÁcter UNIVERSAL DEL aCCESO A LA CULTURA EN INTERNET: UN ANÁlisis DESDE EL PRISMA INTERNACIONAL Y LA EXPERIENCIA DEL ORDENAMIENTO JURÍDICO CUBANO 344 Janny Carrasco Medina

Direito Humanitário e Arte

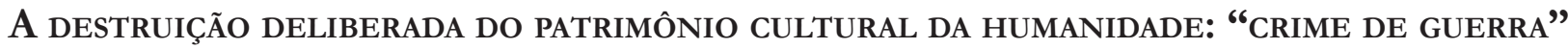
OU "CRIME CONTRA A HUMANIDADE"? Juliette Robichez

Protection OF CULTURAL PROPERTY UNDER INTERNATIONAL HUMANITARIAN LAW: EMERGING TRENDS

Niteesh Kumar Upadhyay e Mahak Rathee

Direito do Mar/Marítimo e Arte.

The underwater Cultural heritage Regime: SOME PROBlems AND POSSible SOlutions. 412 Elina Moustaira

El ROL DEL DERECHO EN LA CONSTRUCCIÓN DEL PATRIMONIO CULTURAL SUBACUÁTICO: APRECiaciones a partirdel estudio del CASo de la Corbeta Inglesa SwifT en Argentina .. 424 Norma Elizabeth Levrand e Nadia Bressan Bernhardt 
INDIGENOUS REFUGEES AND CULTURAL EROSION: POSSIBILITIES AND LIMITS OF INTERNATIONAL REFUGEE AND INDIGENOUS PEOPLES LAW IN THE PROTECTION OF INDIGENOUS CULTURAL EXPRESSIONS RELATED TO TRADITIONAL LAND AND NATIVE LANGUAGE. .440 Rickson Rios Figueira

O RETRATO DE EDMOND BELAMY E A INTERFACE ENTRE ARTE E INTELIGENCIA ARTIFICIAL: POR UMA NOVA DEFINIÇÃo DE AUTORIA E DIREITOS DE PROPRIEDADE INTELECTUAL

Marla Meneses do Amaral Leite Mangiolardo, Patrícia Silva de Almeida e Jonathan Barros Vita

Argumentative aspects of Declaration on the Importance and Value of Universal Museums (2002) 479

Agnieszka Plata

A DestinaÇão dos bens CUlturais EM PROCESSOS PENAIS: A ARTE COMO REPARAÇÃo COLETIVA 488

Inês Virgínia Prado Soares e Otavio Venturini

A Justiça de Pieter Bruegel: direito, violência e a venda nos (nossos) olhos. .501 Rafael Lazzarotto Simioni e Cícero Krupp

Artigos Sobre outros temas

DEVERES INTERNACIONAIS E OBRIGAÇÕES SOCIOAMBIENTAIS PARA EMPRESAS MULTI E TRANSNACIONAIS

Luísa Cortat Simonetti Gonçalves e Adriano Sant'Ana Pedra

Maternidade por substituição: perspectivas da ConferênCia da Haia e suas potenciais INFLUÊNCIAS NO REGRAMENTO BRASILEIRO

Tatiana de A. F. R. Cardoso Squeff e Fernanda Rezende Martins

EL (LARGO) CAMINO DE RECONOCIMIENTO Y EJECUCIÓN DE LAUDOS ARBITRALES DE INVERSIÓN

Thiago Paluma, Ivette Esis e Gabriel Briceño 
A INTERPRETAÇÃo EVOLUTIVA DA CONVENÇÃo AMERICANA SOBRE DiREITOS HUMANOS: UMA REVISÃO DOCUMENTAL DO PERÍODO 1988-2018

Breno Baía Magalhães

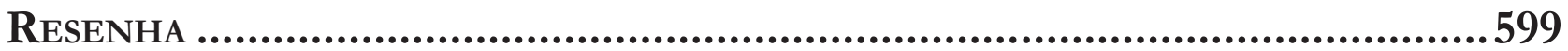

Autonomous Weapons Systems and InTERnATIONAL LAw: A STUDY ON HUMAN-MACHINE INTERACTIONS IN ETHICALLY AND LEGALLY SENSITIVE DOMAINS

Aziz Tuffi Saliba e Lutiana Valadares Fernandes Barbosa 


\title{
A propósito del carácter universal del acceso a la cultura en internet: un análisis desde el prisma internacional y la experiencia del ordenamiento jurídico cubano*
}

\author{
About the universal character of access to \\ culture on the internet.: an analysis from the \\ international perspective and the experience of \\ the Cuban legal system
}

Janny Carrasco Medina**

\section{Resumen}

El reconocimiento de los derechos económicos sociales y culturales (DESC) y específicamente el acceso a internet es un elemento indispensable en la concreción del acceso a la cultura, a través de la responsabilidad adjudicada a los Estados parte como es el caso de Cuba. El objetivo es analizar la concepción internacional de los DESC y específicamente el acceso a la cultura desde la realidad de internet, exponiendo sus desafíos y oportunidades, como mecanismo necesario para incrementar las libertades fundamentales en el contexto cubano. Para ello definimos como la hipótesis del estudio que: el acceso a la cultura en internet en la realidad cubana constituye un ejemplo de la necesidad de reinterpretar los DESC desde el papel de las nuevas tecnologías en la materialización de estos derechos. La metodología empleada es de enfoque transdisciplinar y para ello se apoya en los métodos lógicos, histórico-sociales, culturales y jurídicos, suponiendo la combinación de métodos de las ciencias sociales y jurídicas en particular. Como resultado se concluye que la ampliación e inclusión del acceso a internet como derecho humano, es esencial en la materialización del acceso a la cultura en el contexto de Cuba ya que permitirá proteger los valores culturales locales e incrementar el desarrollo social, aumentando el bienestar del ser humano y garantizar las libertades fundamentales. El aporte del estudio radica en una nueva interpretación de los DESC delante de la presencia de internet en la vida social del individuo en el contexto de la brecha digital cubana.

Recebido em 24/04/2020

Aprovado em 25/03/2021

** Alumna de Pos-Doctorado de la Universidad de Brasilia. Profesora de Pós-Graduación de la Pontificia Universidad Católica de Minas. Dra en Derecho Internacional por la Universidad de Brasilia en 2018. Master en Educación Superior por la Universidad Marta Abreu Las Villas, Cuba en 2010. Licenciada en Derecho por la Universidad Marta Abreu Las Villas, Cuba en 2007. Profesora Asistente de la Universidad Marta Abreu Las Villas, Cuba de 2007 2014.

E-mail: jannycarrasco83@gmail.com
Palabras clave: Derecho internacional. Acceso a la cultura. Internet. Derechos humanos.

\section{Abstract}

The recognition of economic, social and cultural rights (ESCR) and specifically access to the internet is an indispensable element in the realization of access to culture, through the responsibility assigned to the States parties, as is the case of Cuba. The objective is to analyze the international conception of ESCR 
and specifically access to culture from the reality of the internet, exposing its challenges and opportunities, as a necessary mechanism to increase fundamental freedoms in the Cuban context. For this, we define as the hypothesis of the study that: access to culture on the Internet in the Cuban reality constitutes an example of the need to reinterpret ESCR from the role of new technologies in the materialization of these rights. The methodology used is of a transdisciplinary approach and for this it relies on logical, historical-social, cultural and legal methods, assuming the combination of methods from the social and legal sciences in particular. As a result, it is concluded that the expansion and inclusion of internet access as a human right is essential in the materialization of access to culture in the context of Cuba since it will allow protecting local cultural values and increasing social development, increasing the well-being of the population. be human and guarantee fundamental freedoms. The study's contribution lies in a new interpretation of ESCR in light of the presence of the internet in the social life of the individual in the context of the Cuban digital divide.

Keywords: International law. Access to culture. Internet. Human rights.

\section{Introducción}

En el siglo XXI las tecnologías digitales han transformado la esencia de la escena cultural global. En efecto el nuevo escenario de la cultura ha revolucionado industrias enteras, siendo un proceso mundial que ha afectado al Norte y al Sur.

En el contexto del derecho internacional, esta cuestión ha sido objeto de un estudio pormenorizado ya sea en la Declaración Universal de Derechos Humanos de 1948, en el Pacto Internacional de Derechos Económicos Sociales y Culturales de 1966(PIDESC); como en la Convención sobre la Protección y Promoción de la Diversidad Cultural de 2005. Todos estos instrumentos han incorporado de manera progresiva la problemática digital y específicamente el tema del acceso a la cultural en el espacio virtual. Lo que evidencia la significación del tema en el ámbito internacional.

Desde el año 1998 Cuba ha desarrollado un proceso de informatización de la sociedad. Ya en el año 2011 se inició una actualización del modelo económico que enfatiza en la necesidad de contar con una infraestructura tecnológica que permita el desarrollo social (PCC, 2011, p. 17). En este documento se hace referencia a la cultura como un elemento fundamental junto al progreso tecnológico para lograr el desarrollo ${ }^{1}$.

La problemática de un concepto relativo a los DESC ocupa sin lugar a duda, una de las cuestiones que actualmente provocan mayor interés en gran parte de la comunidad académica dedicada a los estudios jurídicosinternacionales. En este sentido, desde nuestro punto de vista, los internacionalistas reiteradamente han pretendido no en pocas ocasiones, darles una aplicabilidad a estos derechos fundamentales siempre desde una visión (reducida) estrictamente técnico-jurídica.

Esta conceptualización, está enfocada sin duda a la búsqueda definitiva de los contornos técnicos jurídicos que ansían precisión en su aplicabilidad y percepción; sin ello resultaría difícil la reivindicación, su goce y ejercicio pleno por parte de los titulares.

Sin embargo, son necesarios nuevos estudios al respecto de los DESC, delante de la realidad tecnológica que impone nuevas maneras de pensar el derecho y sus escopos. Las apreciaciones multidimensionales, arrojan y evidencian la necesidad de respuestas desde la positivación de estos derechos, como es el caso del acceso a internet y el acceso a la cultura en el contexto de Cuba.

El reconocimiento de estos como un conjunto de garantías fundamentales de protección, rebaza toda certidumbre basada exclusivamente en el derecho, pues se tratan de pilares básicos sobre los que se erige toda forma de sociedad contemporánea. En este sentido, el debate en el espacio académico-jurídico en torno a esta realidad camina por interminables laberintos cuando se pretende reconocer el carácter fundamental de los DESC y específicamente el acceso a internet como mecanismo de materialización del acceso a la cultura. De esta manera, esta pretensión encuentra obstáculos difícilmente evitables y que recaen en el valor jurídico de los DESC, desconociendo que los mismos deben concebirse de manera multidisciplinar para la concreción plena e integral de la dignidad humana.

Por otra parte, desde el punto de vista del imprescindible análisis jurídico la normativa de los DESC en

PCC. Lineamientos de la politica económica y social del partido y la revolución para el período 2016-2021. 2017. Disponible en: http://media.cubadebate.cu/wp-content/uploads/2017/07/PDF-321.pdf Acceso en: 31 out. 2020. p. 17. 
el espacio internacional suscita un interés especial desde la realidad cubana, toda vez que aún está pendiente en la doctrina un desarrollo teórico alrededor de ellos, desde la presencia de internet y el enfoque multidisciplinar, radicando así la principal novedad del estudio. Debe recordarse que la construcción teórica de los DESC (acceso a internet y acceso a la cultura), no se agota en el reconocimiento formal de los mismos vía derecho constitucional e infra-constitucional, sino que, por el contrario, parte de un análisis para extenderse a los horizontes del ámbito social donde se materializan.

Tal panorama exhibido por los estudios jurídicos aún está pendiente de resolución definitiva por lo que el caso cubano, evidencia la realidad de las sociedades latinoamericanas, inmersas en una eterna agonía de deuda económica y social que en ocasiones implica un retroceso en la concreción de estos derechos.

Para ello definimos como la hipótesis del estudio que: el acceso a la cultura en internet en la realidad cubana constituye un ejemplo de la necesidad de reinterpretar los DESC desde el papel de las nuevas tecnologías en la materialización de estos derechos.

Ante esta situación la principal motivación del estudio fue ofrecer un conjunto de argumentos, esbozados en el orden teórico doctrinal con el propósito de exponer la realidad cubana para una mejor ponderación de los DESC desde el acceso a internet y su conexión con el acceso a la cultura. Para ello el estudio se apoya en documentación teórica, estadística e informativa sobre el panorama jurídico social cubano, teniendo en cuenta que esta realidad trasciende el plano local hacia el contexto global de cualquier país,

La metodología empleada aborda un enfoque transdisciplinar con métodos lógicos y elementos históricos-sociológicos, culturales y jurídicos, lo que supone la combinación de métodos de las ciencias sociales en general y de las ciencias jurídicas en particular.

Con ello se pretende coadyuvar una mejor compresión y entendimiento que facilite tanto para investigadores de Derecho como para cualquier individuo la titularidad de estos en toda sociedad y específicamente desde las particularidades del ordenamiento jurídico cubano. En ese sentido se aborda el acceso a la cultura desde el derecho internacional, tomando como referencia los principales instrumentos del tema y de los que Cuba es parte, seguidamente se expone los desafíos de la brecha digital, sus definiciones e interrelación con la materiali- zación de los DESC, para finalmente desdoblar todo el bosquejo teórico en la compleja realidad jurídica cubana.

Como resultado se muestra que la ampliación e inclusión del acceso a internet como derecho humano, es esencial en la materialización del acceso a la cultura en el contexto de Cuba ya que permitirá proteger los valores culturales locales e incrementar el desarrollo social, aumentando el bienestar del ser humano. De ahí que el principal aporte del estudio sea dirigido a entender el acceso a internet y el acceso a la cultura como derechos indispensables en la nueva realidad de los DESC en el ámbito tecnológico global.

\section{Tras los causes del acceso a la cultura}

De acuerdo con la Organización de las Naciones Unidas para la Educación, la Ciencia y la Cultura (UNESCO) entendemos por cultura: "conjunto de rasgos diferenciadores, espirituales, materiales, intelectuales y afectivos que caracterizan una sociedad o a un grupo social que abarca, además de las artes y las letras, los modos de vida, las maneras de vivir juntos, los sistemas de valores, las tradiciones y las creencias"2.

Según Fernández Liesa la cultura no ha recibido un tratamiento autónomo dentro del derecho internacional, sino que este ha sido incluido dentro de otras temáticas como es el caso de los derechos humanos, la propiedad intelectual o el patrimonio cultural ${ }^{3}$.

Esta realidad abstracta y dispersa del derecho de la cultura se viene disipando en el orden internacional ya que cada vez es más frecuente la existencia de regulaciones globales de temas vinculados a la cultura. Un ejemplo de ello son las regulaciones relativas a museos, archivos, patrimonio cultural, bibliotecas, cultura popular tradicional, diversidad cultural. Además, el protagonismo que vienen alcanzado los organismos internacionales como la UNESCO o la Oficina Mundial de la Propiedad Intelectual (OMPI), también evidencia una vuelta de página a los temas culturales.

2 UNESCO. Conferencia Mundial de Politicas Culturales. MONDIACULT. México: UNESCO, 1982. p. 20.

3 LIESA, C. F. Cultura y Derecho Internacional. Alcalá: Universidad de Alcalá, 2013. p. 31 
Los tratados internacionales juegan un papel fundamental en la regulación de los derechos culturales. Ello permite instaurar una correlación jerárquica de manera vertical y descendente con las leyes domésticas, estableciendo los preceptos más generales que intervienen en esta relación.

Según González existen dos premisas fundamentales para que el acceso a la cultura sea efectivo: (1) una manifestación de las personas, reflejada en el deseo de acceder a contenidos culturales; cuando las personas acuden a una biblioteca, exposiciones, museos, conferencia, universidad, participan en cualquier manifestación cultural; y (2) las entidades públicas o privada que proporcionan el material o los contenidos culturales, aquellas entidades cuya finalidad es proporcionar la difusión de cultura ${ }^{4}$.

En el caso de la realidad de internet estos dos elementos se entremezclan debido a la ubicuidad, inmediatez y transnacionalidad del medio, lo que exige la mirada del derecho internacional ya que no es un asunto que pueda ser tratado desde la perspectiva local pues tendrá impacto a escala mundial.

Según Rubio, el sistema internacional de derechos humanos experimenta inclusiones abstractas desde exclusiones concretas, lo que denota en la existencia de un derecho internacional poco eficaz; desde el punto de vista de la materialización concreta de estos derechos como es el caso de los derechos culturales ${ }^{5}$.

Con relación a los derechos culturales y coincidiendo con Patricie Bisch los mismos tienen una gran laguna en el derecho internacional de los derechos humanos. Al mismo tiempo Stavenhgen, Francioni o Stamatopoulou también destacan la falta de elaboración conceptual de estos ${ }^{6}$.

Está ambigüedad normativa es lo que posibilita en la esfera internacional una dispersión jurídica que emana hacia dentro del ordenamiento interno de los Estados parte, como exclusiones concretas a las que hace referencia Rubio?.

GONZÁLEZ, M. D. C. B. El derecho de autor y el derecho de acceso a la cultura. Barcelona: Universidad de Barcelona, 1997. p. 180.

5 RUBIO, D. S. Derechos humanos, no colonialidad y otras luchas por la dignidad: una mirada parcial y situada. Campo Jurídico, v. 3, n. 1, p. 181-213, mayo 2015. p.184

6 BISCH, P. M. Definir les droits culturels. Ginebra: UNESCO, 2010. p. 13.

RUBIO, D. S. Derechos humanos, no colonialidad y otras luchas por la dignidad: una mirada parcial y situada. Campo Jurídico, v. 3, n.
Básicamente existen tres instrumentos internacionales de los que Cuba es signatario y que aborda el derecho de acceso a la cultura, ellos son: la Declaración Universal de los Derechos Humanos de 1948, que define en su artículo $\mathrm{n}^{\circ} 19$ el derecho de cada individuo a la libertad de opinión y de expresión, así como el derecho de investigar y recibir; lo que define la presencia del derecho de acceso. Ya el artículo 27.1 define el derecho a tomar parte libremente de la vida cultural de la comunidad, gozar de las artes y participar del progreso científico. La llegada PIDESC, viene a complementar de manera significativa el artículo, 27 de la Declaración Universal. El 15.b) logra enriquecer el acceso a la cultura de manera más clara, reconociéndose el acceso, goce, beneficio y disfrute de los bienes culturales a favor de los ciudadanos. ${ }^{8}$

En este sentido, ya la propia, Observación General n. 25 de 2020 del PIDESC, relativa a la ciencia y los derechos económicos, sociales y culturales, resalta varios criterios a seguir por parte de los Estados signatarios, para el cumplimiento efectivo de los derechos en el consagrados. ${ }^{?}$

Tomando como referencia el artículo 15 b) el referido documento contempla como una necesidad del Estado en la difusión de la ciencia el acceso a internet. Entre las obligaciones subrayadas, destacan: eliminar la censura o las limitaciones arbitrarias al acceso a internet, adoptar medidas legislativas, administrativas, presupuestarias y de otra índole para el pleno disfrute a participar del progreso científico, entre ello facilitar el acceso a internet ${ }^{10}$.

Por último, la Convención sobre la protección y promoción de la diversidad de las expresiones culturales de 20 de octubre de 2005, acentúa el papel de los derechos fundamentales en el desarrollo integral de ser humano; determina la importancia de los derechos culturales el acceso a la cultura en el plano nacional e internacional tal como es abordado en el artículo 2 apartado7. Si bien, este instrumento no alude de manera explícita la cues-

\footnotetext{
1, p. 181-213, mayo 2015.

ORGANIZAÇÃO DAS NAÇÕES UNIDAS. Declaración Universal de los Derechos Humanos. Ginebra: ONU, 1948.

9 ORGANIZAÇÃO DAS NAÇÕES UNIDAS. E/C.12/GC/25. Observación General n. 25, relativa a la ciencia y los derechos económicos, sociales y culturales. Ginebra: ONU, 2020.

10 ORGANIZAÇÃO DAS NAÇÕES UNIDAS. E/C.12/GC/25. Observación General n. 25, relativa a la ciencia y los derechos económicos, so-
} ciales y culturales. Ginebra: ONU, 2020. p. 4-7. 
tión digital, podemos encontrar elementos que nos indican de forma genérica la aplicación de sus postulados en el entorno digital (artículo 4.1).

Tal como refiere Kulesz, la Convención afecta de manera transversal los 4 objetivos primordiales que subyacen en la Convención: flujo equilibrado de bienes y servicios culturales, sostenibilidad de los sistemas de gobernanza para la cultura, integrar la cultura al desarrollo sostenible y promover la libertad de expresión, el derecho a la privacidad y los derechos humanos ${ }^{11}$.

En síntesis, en los instrumentos internacionales abordados, se aprecia que existe una protección de los derechos culturales, entre ellos el acceso a la cultura, pero aún persiste la ambigüedad jurídica que acompaña el tema cultura debido a la complejidad de adaptación del concepto, pues es preciso respetar las particularidades de los Estados que asumen los tratados y la manera en cómo son materializado estos derechos. Además, es evidente la necesidad tanto el ámbito internacional como nacional la interdisciplinariedad del acceso a la cultura en la realidad digital actual.

Para Stavenhagen hay tres concepciones de la cultura en los instrumentos internacionales concebidas como: 1) capital, en cuya acepción el derecho a la cultura sería la igualdad de acceso de todos al capital cultural, al desarrollo cultural; 2) como creatividad, donde el acento se pone en el proceso de creación artística y se mira en los individuos que crean la cultura;3) como modo de vida que es el más común ${ }^{12}$.

La materialización de estas tres concepciones evidencia la necesidad de repensar el acceso a la cultura en el contexto de internet, en la realidad cubana. Debido a la agudización de las brechas digitales, el incremento de la desigualdad y la exclusión del consumo cultural en este espacio, como se profundiza más adelante.

$\mathrm{Al}$ respecto Liesa enfatiza: para que se materialice el derecho a la cultura o el acceso a la cultura, es imprescindible una existencia de triple perspectiva: i). la libertad de creación, y expresión, ii) el reconocimiento de la diversidad creativa; iii). el acceso a los contenidos

\footnotetext{
11 KULESZ, O. El impacto de las tecnologias digitales en la diversidad de las expresiones culturales de España e Hispanoamérica. Paris: UNESCO, 2016. p. 8.

12 STAVENHAGEN, R. Les droit culturels: le point de veu des sciences sociales, pour ou contre les droits culturels? Ginebra: UNESCO, 2000. p. 19-47.
}

culturales ${ }^{13}$.

En síntesis, en los instrumentos internacionales abordados, se aprecia que existe una protección de los derechos culturales relacionada con temas como: lengua, producción cultural y artística, participación en la cultura, patrimonio cultural, y acceso a la cultura; como derecho humano fundamental de manera sucinta.

Ya en el contexto de internet la temática no goza de definiciones precisas que ayuden a entender el comportamiento de este fenómeno en la red. Esta realidad trasciende a la esfera doctrinal en búsqueda de posiciones o definiciones que tampoco enuncian qué y cómo debe ser el acceso a la cultura en internet, correspondiendo a las legislaciones internas resolver esta situación desde las normas sustantivas. Estas carencias propician el incremento de la brecha digital, la minimización de los DESC y por consiguiente un impacto directo en el acceso a la cultura.

\section{Una parada necesaria en la brecha digital}

Como vimos anteriormente la ausencia de definiciones sobre acceso a la cultura y derechos culturales a nivel tratados internacionales, facilita que las nuevas tecnologías sean utilizadas de manera poco eficiente en cuestiones culturales.

Básicamente la brecha digital es entendida como las desigualdades existentes entre el acceso y el uso de las nuevas tecnologías de la información y la comunicación TICs y sobre todo de internet.

De manera suscita Hilbert Martin sostiene que la brecha digital es la que se produce entre aquellos que tienen acceso a las tecnologías digitales y aquellos que no. Hoy en día este fenómeno alcanza no solo a los que tienen o no acceso, sino a los que teniendo acceso no hacen un uso eficiente de ello ${ }^{14}$.

El fenómeno de la brecha digital o la limitación de acceso a internet constituye un elemento de existencia de desigualdad estructural, que se materializa en la esfera del acceso de los individuos a la red y el uso de las

\footnotetext{
13 LIESA, C. F. Cultura y Derecho Internacional. Alcalá: Universidad de Alcalá, 2013. p. 119.

14 HILBERT MARTIN, R. From industrial economics to digital economics. Santiago: CEPAL, 2001. p. 103.
} 
TIC, como parte de su participación en la sociedad del conocimiento y el desarrollo.

De acuerdo con el Informe de la Comisión Económica para América Latina y el Caribe (CEPAL)

la brecha es la línea divisoria entre el grupo de la población que ya tiene la posibilidad de beneficiarse de las TIC y el grupo que aún es incapaz de hacerlo. En otras palabras, es una línea que separa a las personas que ya se comunican y coordinan actividades mediante redes digitales respecto de quienes aún no han alcanzado ese estado avanzado de desarrollo [...] La brecha digital es, en esencia, un subproducto de las brechas socioeconómicas preexistentes ${ }^{15}$.

Ya para el 2019 esta institución, advierte en su informe el impacto de la brecha de capital humano: "el desarrollo de las industrias digitales en un país requiere que las empresas puedan acceder a recursos de inversión, capital humano y capacidad de innovación"16.

Es importante subrayar que desde el año 2012 la Asamblea General de la ONU, a través de su Consejo de Derechos Humanos, registra el acceso a internet como un derecho humano; reconociendo la naturaleza mundial y abierta de este como fuerza impulsora del progreso social. En el mismo documento se exhorta a los Estado a promover y facilitar el acceso a internet como un importante instrumento para el desarrollo y ejercicio de los derechos humanos ${ }^{17}$.

Este posicionamiento de la ONU obliga a los países a adoptar medidas que consigan minimizar la brecha digital a nivel local, posibilitando que las ventajas de la sociedad de la información estén cada vez más disponibles para los usuarios. Esta posición no es un asunto simple de resolver a escala local ya que el problema de la brecha digital tiene una perspectiva tecnológica, económica, social y cultural. El entrecruzamiento de ellos conlleva al fenómeno de la desigualdad digital que evidencian los individuos en el siglo XXI.

La brecha tecnológica supone poder acceder o no a la red, o saber utilizar o no las herramientas digitales. La primera exclusión viene dada por tres cuestiones bá-

15 COMISIÓN ECONÓMICA PARA AMÉRICA LATINA Y EL CARIBE. La sociedad de la información en América Latina y el Caribe. Santiago: CEPAL, 2009. p. 14.

16 COMISIÓN ECONÓMICA PARA AMÉRICA LATINA Y EL CARIBE. Datos, Algoritmos y políticas: la redefinicion del mundo digital. Santiago: CEPAL, 2018. p. 197.

17 ORGANIZAÇÃO DAS NAÇÕES UNIDAS. Consejo de Derechos Humanos. A/HRC/20/L.13. Nueva York: ONU, 2012. sicas: no contar con los medios técnicos y económicos necesarios; no saber qué hacer con la tecnología, cuales beneficios obtener de ella o por no tener interés alguno en ella desde una perspectiva cultural.

En el caso concreto de Cuba resaltamos que ha existido un retroceso marcado por el aspecto económico que ha ido modificando la realidad cubana en temas de acceso digital. Según el informe de la Oficina Nacional de Estadística (ONE) de 2019, en el capítulo 17 dedicado a las Tecnologías de la Información y las Comunicaciones desde el año 2015 no se presta más servicios gratuitos de acceso a internet en los Joven Club de Computación ${ }^{18}$.

La cancelación de este servicio gratuito potencializó en nuestro criterio la brecha digital, cerrando una oportunidad real del acceso a la cultura en el espacio virtual cubano. Aunque la estructura fue mantenida para temas de capacitación digital, la limitación de acceso a internet de manera gratuita dejó sin posibilidades a las personas de menos alcance económico en el país; teniendo a partir de esa fecha que pagar por los servicios de acceso a internet.

La perspectiva económica delimita ese tener o no recursos para adquirir los medios técnicos necesarios para disfrutar de conexión a internet, condicionando así las oportunidades de acceso a la red. Intrínsecamente a ella está la brecha social, determinada por el lugar que ocupes en un estrato social tendrás mayor o menor posibilidades de acceso y harás mejor o peor uso de la tecnología.

Desde la arista cultural las brechas estarán delimitadas por la familiarización del individuo con la tecnología, uso y capacidades y por otro lado, real optimización que pueda hacer en uso, venciendo barreras como el colonialismo lingüístico, saber lidiar con cuestiones de saturación de información, desarrollar capacidades para librarse de intoxicación informativa y elevar los niveles de consumo de contenidos culturales, apartando el mercantilismo y el globalismo cultural que predomina en la red.

Si bien internet es un espacio de libertad, de creación y expresión cultural desde las diferentes redes sociales o plataformas, esto no es aprovechado de manera eficien-

18 ONET. Anuario Nacional de Estadistica 2019. La Habana: ONET, 2019. Disponible en: http://www.onei.gob.cu/ Acceso en: 20 abr. 2020. 
te. La falta de una regulación sobre temas de diversidad creativa y como esta debe ser puesta a disposición del público para el acceso en la red, evidencia que el uso de internet no sea optimizado por la mayoría de los individuos con oportunidades de acceso; lo que va creando enormes lagunas de desigualdad cultural, que se prolongan en el tiempo.

Desde la perspectiva de Gigler y Bjorn opinión que compartimos;

las capacidades que resultan indispensables para el desarrollo de los individuos en la Sociedad de la Información y el conocimiento, la pobreza de comunicación e información (pobreza digital) se constituye como: "la privación de las capacidades básicas de participación en la sociedad de la información'. ${ }^{19}$

Pero para materializar el acceso a la cultura, es esencial la puesta a disposición del público o comunicación de una determinada obra o producto cultural y el consumo de esta por parte de los usuarios según refleja González Buganza, lo que alcanzará significado en la medida en que tenga mayor o menor reconocimiento dentro del universo intelectual o cultural, según sea el caso. Un fenómeno complejísimo en internet por amplísima diversidad de productos culturales y sus públicos, así como por el alcance internacional del mismo ${ }^{20}$.

Un ejemplo de ello es la llamada Cultura 2.0. Este fenómeno socio-tecnológico propio del siglo XXI demuestra la relación entre la tecnología de comunicación desarrollada a través de las redes sociales, que está al alcance de la mayoría de las personas y la crisis económica y de valores. Este proceso ha desencadenado una profunda dicotomía hacia todo lo institucionalizado, creando una sociedad más crítica, participativa, colaborativa que se nutre de la creación de culturas alternativas que circulan fuera del poder cultural establecido. Esta llamada Cultura 2.0 escapa de las instituciones, legislaciones y estructuras preestablecidas que históricamente legitimaban el poder jurídico del desarrollo cultural.

Por tanto, el acceso a la cultura desde el contexto digital trae un reflejo palpable de la brecha digital, siendo la nueva cara de la desigualdad en el siglo XXI que re-

\footnotetext{
19 GIGLER, Björn-Sören; BARJA, Gover. Pobręa digital: las perspectivas de América Latina y el Caribe: qué es y cómo demir la probreza de información y comunicación en el contexto latinoamericano. La Paza: Dirsi, 2009. p. 14.

20 GONZÁLEZ, M. D. C. B. El derecho de autory el derecho de acceso a la cultura. Barcelona: Universidad de Barcelona, 1997. p. 187.
}

fleja el modelo económico del capitalismo globalizado; provocando inequidades entre diferentes grupos sociales en términos del acceso, de capacidades cognitivas y de conocimiento que van a materializarse en el uso de las TIC y de las que Cuba no escapa pese a tener un sistema social que dista mucho del capitalismo en Latinoamérica.

\section{Concretamente el fenómeno cubano}

El avance tecnológico a nivel mundial no ocurre de la misma manera en todos los países, ello va a depender en gran medida de las políticas públicas que incentiven la socialización y el acceso a internet de todos los ciudadanos. Por otro lado, el progreso de la sociedad informacional no se desarrolla a espaldas del acceso al conocimiento y la cultura; ambos elementos deben ir junto para garantizar el pleno acceso a la cultura para todos según lo enuncia el artículo 27 de la Carta de Derechos Humanos de la ONU y de la que Cuba es signataria.

Como fuentes reconocidas del Derecho Internacional, los tratados internacionales juegan un papel determinante en la definición de principios, derechos y obligaciones para los Estados que los suscriben. Partiendo de esta definición es importante delimitar que, en el ámbito cubano, los tratados internacionales tienen un rango infra-constitucional según lo enunciado por el artículo 8, lo que sumado a la falta de un tribunal constitucional deja en total estado de vulnerabilidad el disfrute de derechos fundamentales como el acceso a la cultura o el acceso a internet.

Estos preceptos junto a la politización de los derechos humanos en la legislación cubana acaban frustrando el disfrute de estos derechos, así como demonizando a quien posee u opta por alternativas que salen del marco del sistema social imperante; cuestiones incompatibles con la realidad internacional y que necesitan ser reformuladas de manera clara y objetiva a través de normas jurídicas efectivas.

De Acuerdo com Bentes e Brígida y opinión que compartimos, los DESC de los Estados miembros garantizan la efectividad de los derechos fundamentales para la promoción y aumento de las capacidades humanas, en aras del goce y bienestar de los objetivos indi- 
viduales y sociales ${ }^{21}$. En este sentido la realidad cubana exacerba en su legislación una protección discreta y sobreentendida, sin otorgar mayor tutela jurídica al acceso a internet, lo que repercute en el acceso a la cultura.

Otro elemento el destaque con los tratados internacionales, es que, bajo los preceptos de alejarse de políticas neoliberales y mercantilistas, el derecho de acceso a internet no ha sido considerado como un derecho fundamental. Esta realidad provoca un estado de vulnerabilidad de los usuarios cubanos, en materia de internet pues al no ser consagrado en la Carta Magna, si prerrogativas quedan subordinadas a normas inferiores y cuestiones, definiciones políticas sin protección jurídica efectiva.

Un ejemplo de ellos es la llegada del Decreto-Ley 370/2018 "sobre la informatización de la sociedad cubana" ha dejado varios elementos del ámbito internacional en posiciones difusas o un tanto ambiguas. En su artículo $\mathrm{n}^{\circ} 4 \mathrm{y} \mathrm{n}^{\circ} 5$ se define que el objetivo de la norma es garantizar el derecho de acceso y participación de las personas naturales y jurídicas en la sociedad de la información, sin que existan criterios más específicos sobre que debe ser entendido por acceso y cuáles son las premisas por cumplir para ello. Ya el artículo nº 20 deja en manos del Ministerio de Cultura la definición de temas como derecho de autor, protección del patrimonio nacional.

Entre los principales aportes de la norma está la protección del proceso de informatización de la realidad cubana, con el objetivo de preservar las conquistas sociales, el bienestar de la población y la defensa del Estado Socialista. Este instrumento se presenta como un mecanismo que garantiza la satisfacción de las necesidades en la esfera de la vida social en lo relativo a las tecnologías de la información y las comunicaciones.

Esta norma es un reflejo de la realidad internacional y como ella impacta dentro del ordenamiento interno. La ausencia de definiciones sobre cuestiones de acceso a la cultura, derechos culturales, brechas digitales propician que contextos jurídicos diversos no resuelvan estas lagunas y que emitan instrumentos difusos y poco ajustados al contexto tecnológico global.

\footnotetext{
${ }^{21}$ BENTES, Natalia Mascarenhas Simões; BRÍGIDA, Yasmim Salgado Santa. Vinculação dos direitos econômicos, sociais e culturais: uma discussão do desenvolvimento humano com base no conceito de Amartya Sen sobre o mínimo existencial. Revista de Direito Internacional, v. 15, n. 3, p. 98-120, 2018. p.119.
}

Las bases legislativas para el acceso a internet y por ende el acceso a la cultura en internet en el caso cubano, fueron establecidas de manera sucinta, escueta y con la persistencia de lagunas jurídicas. No es suficiente la existencia de normas para establecer el acceso universal a internet, es necesario la implementación de políticas y medidas que apoyen el acceso a la cultura desde diferentes ámbitos como: la creación de artes digitales, experimentación electrónica y formación de artistas, así como medidas que incentiven el consumo de contenidos electrónicos pagos y gratuitos que fomente valores locales.

De acuerdo con Ruiz, el concepto de cultura, como objeto de estudio del derecho Constitucional, es indeterminado a la vez que es inexcusable, al constituirse como un verdadero reto para los constitucionalistas por su carácter polisémico y elástico ${ }^{22}$.

Sin embargo, si se estudia el texto constitucional cubano, es palpable que el legislador obvió garantizar taxativamente esos derechos, mucho menos explicar su alcance. No obstante, eso no significa que esta no lo tutele de alguna manera.

Lo que sucede es que la protección del acceso a la cultura viene circunscrita a varios otros derechos, como la libertad de expresión, el disfrute de la cultura, el acceso a la enseñanza tal como reconoce el texto constitucional, lo que no sucede con el acceso a internet. Es importante resaltar que en la realidad tecnológica actual la efectiva protección de los DESC necesita de la inclusión del acceso a internet como elemento indispensable, lo que no evidenciamos en el contexto jurídico cubano.

Cuba reconoce como derecho humano fundamental el disfrute de la cultura por todo el pueblo y la preponderación de los derechos sociales por encima de los individuales, según el artículo $n^{\circ} 32$.- El Estado orienta, fomenta y promueve la educación, las ciencias y la cultura en todas sus manifestaciones; así como el artículo $\mathrm{n}^{\circ} 79$ - todas las personas tienen derecho a participar en la vida cultural y artística de la nación. El Estado promueve la cultura y las distintas manifestaciones artísticas, de conformidad con la política cultural y la ley.

Esta protección constitucional garantiza en gran medida que las políticas culturales en Cuba tengan protagonismo como elementos de crecimiento social y cultural de la población, pero fuera del ámbito de internet.

RUIZ, G. R.R. Introduccion metodológica sobre el estudio de la cultura por el derecho constitucional. Valencia: Tirant Lo Blanch, 2005. 
Su objetivo es ampliar la difusión cultural nacional y el consumo consciente; al mismo tiempo que intenta escapar de políticas mercantilistas que dominan los espacios culturales en los sistemas sociales capitalistas.

En Cuba la política cultural está en manos de la representación gubernamental. Tanto el desarrollo de la cultura como el capital cultural son determinados a través de políticas públicas que llevan a cabo las diferentes instituciones culturales. Poco se ha enfatizado en el papel y el lugar que deben ocupar las nuevas realidades creativas que emergen con la abertura de internet.

Una muestra de la materialización del acceso a la cultura en Cuba está dada en las diferentes manifestaciones artísticas que son ofrecidas al público en disimiles espacios culturales, fueran del medio digital. Tal como evidencia el informe de la Oficina Nacional de Estadística de 2018 ${ }^{23}$ (ONET, 1986-2018):

Ilustración 1 - Ofertas Culturales en Cuba en espacios no virtuales

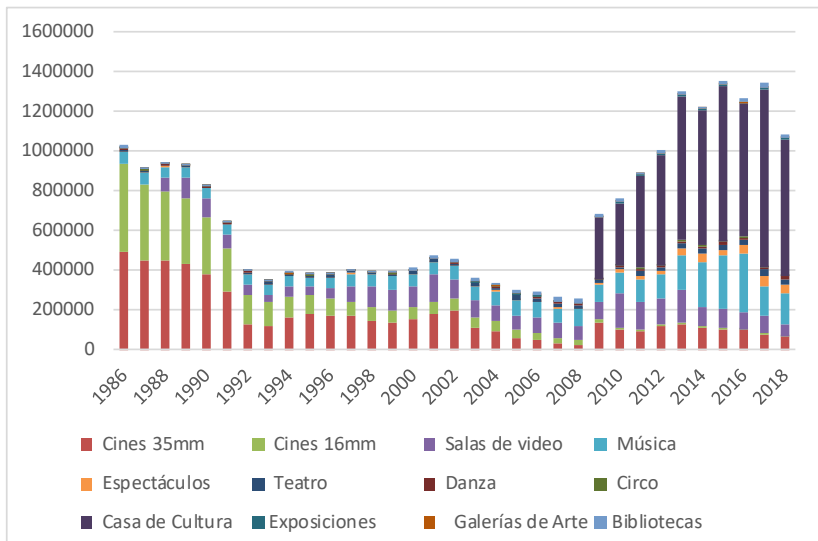

\section{Fonte: ONET}

La lectura de esta estadística evidencia la dimensión pública del acceso a la cultural y la importancia que el Estado concede a las políticas públicas en aras de salvaguardar y preservar la creación artística nacional y el disfrute de la cultura en los diferentes espacios. Acá la materialización del acceso a la cultura es evidente; ya que la diversidad de espacios a disposición de la ciudadanía, son una muestra fehaciente del papel del Estado en garantizar tales derechos. Aunque otra lectura de los mismos datos evidencia como los espacios culturales creados en internet y el acceso a la cultura desde este medio, no son tomados en cuenta como un fenóme-

23 ONET. Manifestaciones artísticas en Cuba ofrecidas a la población. La Habana: ONET, 1986-2018. no que crece en el contexto cubano y que constituye el futuro próximo. Pues aun cuando el acceso a internet existe en el país desde 2000 la promoción cultural en internet no se reporta en este estudio del 2018.

Tal como reflejan estas estadísticas, Cuba presenta una amplia gama de políticas que contribuyen a promover el acceso a la cultura, pero fuera del marco digital. Está claro que la ausencia de las nuevas tecnologías implica un distanciamiento de los medios de distribución digital, como libros, música y cine, imposibilitando que de manera más eficaz puedan ser consumidos productos culturales cubanos desde cualquier lugar del país.

Es evidente que la oferta digital con la que deben competir los productos culturales nacionales es más abundante y estructurada. Entre las principales barreras a superar esta la brecha digital que atenta contra el propio mantenimiento y sobrevivencia de la cultural local, seguido de un divorcio entre la infraestructura de conexión y equipamiento y los espacios culturales.

El derecho de acceso cultural, que es otorgado como un pilar esencial de las políticas públicas cubanas, no corre la misma suerte en el espacio virtual debido a varios factores: la baja proporción de conexión de la población versus altos costos, el poco soporte de la tecnología y la mercantilización. Como parte de un fenómeno global que es internet se importa esa manera de consumo de cultura virtual que pasa necesariamente por el protagonismo de las redes sociales y que trae nuevos significados a la concepción de cultura de los instrumentos internacionales enunciadas con anterioridad por Stavenhagen ${ }^{24}$.

Por ejemplo, la conectividad de la población según informaciones de la prensa oficial en la isla, emitidas por la Empresa de Telefonía (ETECSA), al cierre de 2019 existían en la isla 1.4 millones de teléfonos fijos, 5.7 millones de líneas móviles, 3268 radiobases instaladas, 124000 servicios de Nauta Hogar (internet discada en casa), 1161 salas de navegación, 1034 sitios wifi y 2 millones de cuentas permanentes para navegación por internet ${ }^{25}$.

Téngase en cuenta que la población cubana está compuesta por 11215344 habitantes, distribuidos en ciones en 2019. Granma, La Habana, 2019. p. 2. 
15 provincias con 168 municipios y con un promedio de urbanización del $77 \%$; de acuerdo las cifras oficiales del Ministerio de Salud Pública Datos que, comparados con los anteriores, demuestra la baja proporción de conexión y acceso tecnológico que se tienen en el país. Pues sólo 2 millones de personas (menos de un cuarto del total de 11 millones), poseen cuentas permanentes para navegación por internet, de un total de 5.7 millones que tienen líneas móviles; lo que representa un $82 \%$ de la población nacional sin acceso a internet ${ }^{26}$.

Las condiciones de conexión a internet en Cuba con una velocidad de navegación según datos oficiales de ETECSA, es de 64kbs-155Mgs dependiendo del tipo de conexión y de las condiciones físicas. Aparentemente esta realidad no muestra que el consumo cultural en línea en Cuba es prácticamente imposible, pero si miramos hacia el valor de la conexión por hora, la realidad es otra.

Por ejemplo, los precios para cada tipo de conexión se mantienen de la siguiente forma: la oferta de un paquetes mínimo de datos móviles de 400MB tiene un calor de 125.00cup (según moneda oficial, que por tasa de conversión redondeada equivale a 5 dólares), la hora de conexión en zonas públicas wifi tienen un valor de 25.00cup (valor de cambio redondeado de 1dolar), y el servicio de Nauta Hogar conexión discada desde casa con un valor de 17.50cup por hora de conexión (con valor de 0.70 dólar ${ }^{27}$.

Un cálculo promedio de los costos de conexión mensual, y según los diferentes tipos conexión a internet que se ofertan serían: paquete mínimo de datos móviles de 400MB por semana tendría un valor 500.00cup (equivalente a 20 dólares); una 1 hora de conexión de zonas públicas de wifi sería 750.00cup (equivalente a 30 dólares) y servicio de Nauta Hogar conexión discada desde casa sería 525.00cup (equivalente a 21 dólares). Estos precios demuestran que el consumo mínimo diario de 1 hora de conexión, para un cubano medio es costoso, casi la mitad de un salario medio y más de la mitad de un salario mínimo promedios; pues la media salarial ronda los 1067.00cup (que equivale a un total redondeado de 40.00 dólares $)^{28}$.

26 CUBA. Ministerio de Salud Pública. Anuario Estadístico de Salud 2018. La Habana: Ministerio de Salud Pública, 2019. p. 11.

27 ETECSA. Preguntas más frecuentes. Disponible en: http://www. etecsa.cu/internet_conectividad/pmf/. Acceso en: 20 abr. 2020.

28 GRANMA. Alejandro Gil: La economía cubana no debe decrecer en 2019. Disponible en: http://www.granma.cu/cuba/2019-12-20/
Hasta el momento el foco del gobierno cubano ha estado en la infraestructura técnica y precios que permitan sustentar las inversiones, pero que acaban limitando el acceso, pues dejan de considerar que la inclusión digital del acceso a la cultura de productos cubanos es una necesidad. De persistir esta realidad Cuba corre el riesgo de transformar sus usuarios en consumidores de productos y aplicaciones de contenido generado desde fuera del territorio nacional, lo que conduce al fomento de un fenómeno negativo en los términos de empleo, y protección de la cultural local; facilitando un mayor espacio para los conglomerados culturases como Youtube, Facebook, Spotify. Esta realidad cuestiona la sobrevivencia de la diversidad de las redes digitales y la preservación de la cultural local y su acceso.

Según el Informe de la Unión Internacional de Telecomunicaciones para el 2018 Cuba estaría en el lugar 137 de 176 a nivel global en términos de acceso a internet, lo que evidencia no solo la ineficacia y el poco desarrollo tecnológico al respecto en el territorio. Este informe también hace referencia a que muchos datos relacionados con el acceso no pueden ser procesados porque no están disponibles, evidenciándose el control estatal sobre las informaciones referentes a conexiones digitales en el país ${ }^{29}$.

Otro elemento que resalta en la realidad cubana es la mercantilización de la cultura en internet. Si bien es un fenómeno propio del capitalismo globalizado e interconectado, ha penetrado en los más diversos sistemas sociales como el cubano. Al respecto, datos del sitio Statcounter GlobalStats apuntan que en el año 2019 las principales redes sociales utilizadas por la población en la isla fueron: Facebook 71,6\%; Pinterest 10,92\%; Instagram 7,63\%; YouTube 4,91\%, Twitter 4,83\% y Tumblr 0,08\%. (la última no se cual es)

Tomando ejemplo de manera aleatoria, la página oficial de algunas instituciones culturales cubanas en Facebook durante el 2019, se evidencia la desproporcionalidad entre el número de usuarios registrados en ETECSA (un aproximado de 2 millones de cuentas permanentes para navegación por internet) y el de seguidores de estas. Tal como muestra la figura $\mathrm{n}^{\mathrm{o}} 2$.

alejandro-gil-la-economia-cubana-no-debe-decrecer-en-2020. Acceso em: 20 abr. 2020.

29 UIT. Statistical reports. Measuring the information Society Report. 2018. Disponible en: https://www.itu.int/en/ITU-D/Statistics/ Pages/stat/default.aspx Acceso em: 20 abr. 2020. 
Ilustración 2 - Páginas de Facebook de las principales Instituciones Culturales en Cuba

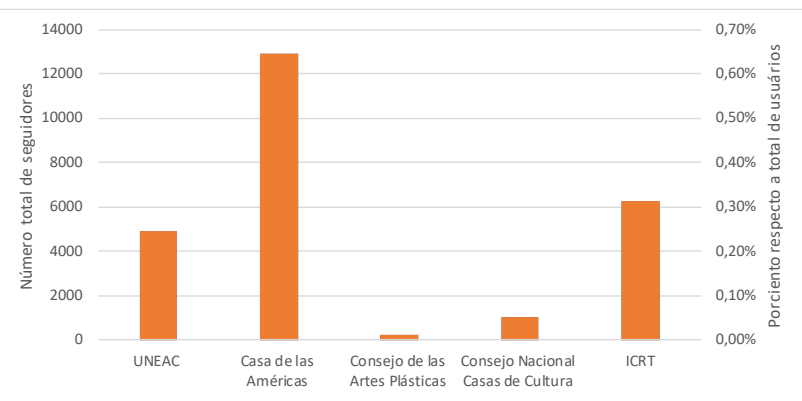

Fonte: ETECSA

Es evidente que la bajísima proporción de usuarios en estas instituciones son el resultado del impacto que tiene la brecha digital en el acceso a la cultura y la preservación de los productos culturales locales. El empleo de los usuarios de las redes sociales está volcado a la utilización como mecanismos de comunicación y socialización. Se evidencia además que el consumo de productos artístico-culturales en este escenario posee un mayor desplazamiento hacia los escenarios offline.

$\mathrm{Al}$ igual que en otras realidades la cultura 2.0 en el caso de Cuba, también va ganado espacio en el desarrollo socio-tecnológico. El protagonismo de las redes sociales, el incremento de la relación tecnología y comunicación, y el divorcio con el poder institucionalizado también son fenómenos que están presentes en el contexto digital cubano. Es evidente que la sociedad cubana viene transformándose y abrazando dinámicas más críticas, participativas y colaborativas desde el ambiente digital; confluyendo así en la aparición de nuevos espacios culturales fuera de las instituciones estatales.

Es pertinente resaltar, que la ausencia de relaciones diplomáticas entre Cuba y Estados Unidos ha tenido un impacto directo en este tema. No solo por las limitaciones de acceso a internet, sino por la vulnerabilidad que acarrea el uso de las redes sociales a los usuarios cubanos. En cualquier escenario global estas empresas poseen en lo mínimo representaciones en los países donde operan, pero en el caso de Cuba esta ausencia es persistente.

Esta particularidad del fenómeno cubano evidencia la necesidad de un papel protagónico, proactivo y eficaz del acceso a la cultura en internet por parte del Estado. Reforzar su protección en el plano domestico minimiza el desamparo en el que se encuentra no solo los usuarios sino las producciones culturales cubanas en estas plataformas.
Es importante resaltar que el uso de las redes sociales en Cuba también contribuye a la creación de una nueva diversidad cultural creativa. Su protagonismo trae nuevos significados al papel cultura popular tradicional y como esta se adapta a la contextualización tecnológica. La concepción del concepto de cultura enunciado por la UNESCO reafirma que existe y está siendo creada una cultura digital reflejo de las condiciones sociales y tecnológicas del siglo XXI.

Resignificando las palabras de Stavenhagen ${ }^{30}$, Cuba es un ejemplo de la transformación de los preceptos que definen el concepto de cultura en el plano internacional. El capital y la creatividad ya escapan hacia una realidad diferente de las establecidas por las instituciones culturales, son parte del reflejo de los nuevos modos de vida que trae internet.

Viendo a internet como un artefacto de producción y consumo cultural de acuerdo con el concepto de cultura ofrecido por la UNESCO, debemos entender que es un fenómeno inmerso en un contexto social determinado. Internet por sí sola no genera cultura, las construcciones culturales y el acceso a ellas se dan de la mano de las experiencias subjetivas de los usuarios apoyadas en estructuras lógica y económicas; reflejando los diferentes sistemas en que vivimos y actuamos.

Cuba es un reflejo de la importancia de otorgar protección jurídica al acceso a internet y su relación con los derechos humanos desde el ámbito internacional, pues de no existir estos compromisos internacionales, el plano de estos derechos quedaría mitigado a la voluntad del legislador local. Aun cuando la creación de instrumentos internacionales es desde una perspectiva top-down, la mera existencia de tratados o recomendaciones ya coloca en los Estados participantes la responsabilidad de garantizar estándares mínimos de protección tal como refleja el caso cubano.

\section{Consideraciones Finales}

Durante el desarrollo de este estudio: la principal problemática sobre el acceso a internet y el acceso a la cultura en el contexto cubano, está fundamentada

30 STAVENHAGEN, R. Les droit culturels: le point de veu des sciences sociales, pour ou contre les droits culturels? Ginebra: UNESCO, 2000. p. 19-47. 
de manera genérica como carácter fundamental de los DESC, al mismo tiempo que se evidencia poca construcción doctrinal en torno a estos derechos en el ámbito digital. Esta realidad evidencia que la positivación a que han sido sometido estos derechos no es suficiente para garantizar su materialización.

En este sentido debemos limitar que los DESC representan un amplio catálogo de exigencias para los Estados parte en la consecución de la dignidad de la persona humana y las garantías fundamentales, que impone el mayor cuidado y protección a cualquier organización jurídico-política. Por tanto, su eficacia jurídica dependerá de las concretas posibilidades reales de ejercicio y protección jurisdiccional que cada Estado le otorgue en su ordenamiento jurídico.

Otro aspecto para resaltar es la concreción material del acceso a internet y el acceso a la cultura, depende directamente de las condiciones sociales, económicas y políticas que cada sociedad brinda. Por lo que, en el caso cubano, la carencia de estas o su difusa protección, otorgan una incapacidad de realización plena de estos derechos.

Superar la brecha digital que se materializa en el acceso a la cultura y sus disimiles facetas, es uno de los principales desafíos a resolver en el caso cubano, donde urge revisar y en su caso reformular las estrategias políticas y las normas existentes al respecto, partiendo del protagonismo de las TIC en el desarrollo social y cultural y en la concreción de los DESC en el siglo XXI.

La irreversibilidad de estos derechos en la realidad cubana debe ser apoyada en tres grandes acciones: consolidar el acceso a internet como un derecho fundamental de manera explícita, utilizar la infraestructura existente para potencializar el acceso a la cultura en internet; llevar a cabo una cruzada nacional en favor de la cultura nacional en las rede sociales, ampliando las políticas públicas que favorezcan la materialización de estos derechos (acceso a internet y acceso a la cultura) de manera efectiva. En resumen, el acceso a la cultura en internet en el contexto de internet en Cuba debe convertirse en una prioridad jurídica y social.

Finalmente, se reafirma que el acceso a internet cambió la materialidad de estos derechos (DESC) y más específicamente el acceso a la cultura, lo que conlleva de modo irrefutable que las futuras normas cubanas o en otros contextos jurídicos, en temas de derechos culturales y acceso a internet, sean pensada desde esta realidad.
No podemos hablar de acceso a la cultura sin la presencia de internet en el siglo XXI.

\section{Referencias}

ALMEIDA, D. Un tribunal internacional para internet. São Paulo: Almedina, 2015.

ALONSO, G. M. Alfabetización digital múltiple: algo más que ratones y teclas. Barcelona: Gedisa, 2003.

BENTES, Natalia Mascarenhas Simões; BRÍGIDA, Yasmim Salgado Santa. Vinculação dos direitos econômicos, sociais e culturais: uma discussão do desenvolvimento humano com base no conceito de Amartya Sen sobre o mínimo existencial. Revista de Direito Internacional, v. 15, n. 3, p. 98-120, 2018.

BISCH, P. M. Definir les droits culturels. Ginebra: UNESCO, 2010.

CALDUCH CERVERA, R. Conflictos internacionales culturales y violencia terrorista: derechos humanos y conflictos internacionales. Bilbao: Servicios Editoriales del País Vasco, 2006. Disponible en: https://www.ucm.es/ data/cont/media/www/pag-55164/9cursvitoria.pdf Acceso en: 31 out. 2020.

CANCLINI, N. G. Desiguales y desconectados. Barcelona: Gedisa, 2006.

CEES, H. Digital Fatso en Mensenrechten in cyberspace. Amsterdan: Boom, 1999.

COMISIÓN ECONÓMICA PARA AMÉRICA LATINA Y EL CARIBE. Datos, Algoritmos y politicas: la redefinicion del mundo digital. Santiago: CEPAL, 2018.

COMISIÓN ECONÓMICA PARA AMÉRICA LATINA Y EL CARIBE. La sociedad de la información en América Latina y el Caribe. Santiago: CEPAL, 2009.

CUBA. Constitución de la República de Cuba. La Habana: Gaceta Oficial de la República de Cuba, 2019.

CUBA. Decreto Ley 370/2018 sobre la informatización de la sociedad cubana. La Habana: Consejo de Estado, 2018.

CUBA. Ministerio de Salud Pública. Anuario Estadístico de Salud 2018. La Habana: Ministerio de Salud Pública, 2019.

CUBA. Resolución 127/2007 sobre el Sistema Informático. La Habana: Gaceta Oficial de la República de Cuba, 2007. v. 1. 
DIMAGGIO, Paul et al. Social implications of the internet. Annual Review of Sociology, v.27, p. 307-336, 2001.

GIGLER, Björn-Sören; BARJA, Gover. Pobreza digital: las perspectivas de América Latina y el Caribe: qué es y cómo demir la probreza de información y comunicación en el contexto latinoamericano. La Paza: Dirsi, 2009.

GONZÁLEZ, M. D. C. B. El derecho de autory el derecho de acceso a la cultura. Barcelona: Universidad de Barcelona, 1997.

HILBERT MARTIN, R. From industrial economics to digital economics. Santiago de Chile: CEPAL, 2001.

UIT. Statistical reports. Measuring the information Society Report. 2018. Disponible en: https://www.itu.int/en/ ITU-D/Statistics/Pages/stat/default.aspx Acceso em: 20 abr. 2020.

KULESZ, O. El impacto de las tecnologias digitales en la diversidad de las expresiones culturales de España e Hispanoamérica. Paris: UNESCO, 2016.

LIESA, C. F. Cultura y Derecho Internacional. Alcalá: Universidad de Alcalá, 2013.

MENDOZA-RUANO, J. J.; CALDERA-SERRANO, J. Umbrales para la determinación de la brecha digital: comparativa entre regiones desarrolladas. Transinformação, Campinas, v. 26, p. 125-132, ago. 2014.

MORENO, I. Globalizacion y cultura, cultura y globalización: entre el conflicto y el dialogo. Alicante: Publicaciones de la Universidad de Alicante, 2005.

MUNIZ, S. A verdade seduzida: por um conceito de culturna no Brasil. Rio de Janeiro: Renovar, 1988.

ONET. Manifestaciones artísticas en Cuba ofrecidas a la población. La Habana: ONET, 1986-2018.

ORGANIZAÇÃO DAS NAÇÕES UNIDAS. Consejo de Derechos Humanos. A/HRC/20/L.13. Nueva York: ONU, 2012.
ORGANIZAÇÃO DAS NAÇÕES UNIDAS. Declaración Universal de los Derechos Humanos. Ginebra: ONU, 1948.

ORGANIZAÇÃO DAS NAÇÕES UNIDAS. E/C.12/ GC/25. Observación General n. 25, relativa a la ciencia y los derechos económicos, sociales y culturales. Ginebra: ONU, 2020 .

ORGANIZAÇÃO DAS NAÇÕES UNIDAS. Pacto Internacional de los Derechos Económicos, Sociales y Culturales. Ginebra: ONU, 1966.

PCC. Lineamientos de la política económica y social del partido y la revolución para el periodo 2016-2021. 2017. Disponible en: http://media.cubadebate.cu/wp-content/uploads/2017/07/PDF-321.pdf Acceso en: 31 out. 2020.

PEÑA, O. A. P. Protección de las obras de la cultura popular tradicional por el derecho de autor: especial referencia a Cuba. La Habana: Abril, 2010.

RAJAGODAL, B. El derecho internacional desde abajo. Bogotá: Isla, 2005.

REYGADAS, L. La apropiación: destejiendo las redes de la desigualdad. Barcelona: Anthropos, 2008.

RODRIGUEZ, S. A. Servicio de internet en Cuba actualizaciones en 2019. Granma, La Habana, 2019.

RUBIO, D. S. Derechos humanos, no colonialidad y otras luchas por la dignidad: una mirada parcial y situada. Campo Jurídico, v. 3, n. 1, p. 181-213, mayo 2015.

RUIZ, G. R.R. Introduccion metodológica sobre el estudio de la cultura por el derecho constitucional. Valencia: Tirant Lo Blanch, 2005.

STAVENHAGEN, R. Les droit culturels: le point de veu des sciences sociales, pour ou contre les droits culturels? Ginebra: UNESCO, 2000.

UNESCO. Conferencia Mundial de Politicas Culturales. MONDIACULT. México: UNESCO, 1982.

UNESCO. Convención Universal sobre Protección y Promoción de la Diversidad Cultural. Paris: Unesco, 2005. 
Para publicar na Revista de Direito Internacional, acesse o endereço eletrônico www.rdi.uniceub.br ou www.brazilianjournal.org.

Observe as normas de publicação, para facilitar e agilizar o trabalho de edição. 